\title{
Brain white matter fibre tracts: a review of functional neuro-oncological relevance
}

\author{
Natalie L Voets, ${ }^{1,2}$ Andreas Bartsch, ${ }^{3}$ Puneet Plaha ${ }^{1,2}$
}

${ }^{1}$ Nuffield Department of Clinical Neurosciences, University of Oxford, John Radcliffe Hospital, Oxford, UK

${ }^{2}$ Department of Neurosurgery, John Radcliffe Hospital, Oxford University Hospitals NHS Foundation Trust, Oxford, UK ${ }^{3}$ Department of Neuroradiology, University of Heidelberg, Heidelberg, Germany

Correspondence to Dr Natalie L Voets, FMRIB Centre, University of Oxford, John Radcliffe Hospital, Headington, OX3 9DU, Oxford natalie.voets@ndcn.ox.ac.uk

Received 31 March 2017 Revised 3 June 2017 Accepted 20 June 2017 Published Online First 14 July 2017

\section{CrossMark}

To cite: Voets NL, Bartsch A, Plaha P. J Neurol Neurosurg Psychiatry

2017:88:1017-1025.

\section{ABSTRACT}

State-of-the-art glioma treatment aims to maximise neuro-oncological benefit while minimising losses in quality of life. Optimising this balance remains hindered by our still limited understanding of information processing in the human brain. To help understand individual differences in functional outcomes following neuro-oncological treatment, we review mounting evidence demonstrating the fundamental role that white matter connections play in complex human behaviour. We focus on selected fibre tracts whose destruction is recognised to elicit predictable behavioural deficits and consider specific indications for non-invasive diffusion MRI tractography, the only existing method to map these fibre tracts in vivo, in the selection and planning of neuro-oncological treatments. Despite remaining challenges, longitudinal tract imaging, in combination with intraoperative testing and neuropsychological evaluation, offers unique opportunities to refine our understanding of human brain organisation in the quest to predict and ultimately reduce the quality of life burden of both surgical and non-surgical first-line neurooncological therapies.

\section{RELEVANCE OF WHITE MATTER IN NEURO-ONCOLOGICAL TREATMENT}

Mounting evidence suggests that preservation of long-range white matter (WM) fibre bundles is a key determinant of functional outcomes after adult neurosurgical ${ }^{1}$ oncology treatment. Maximal surgical resection remains the first-line treatment for high-grade brain tumours, and in low-grade gliomas, it offers opportunities to dramatically prolong life expectancy. ${ }^{2}$ When attempting maximal resections, understanding which fibre bundles mediate specialised information processing is emerging as increasingly important to tailor treatment to long-term quality of life. ${ }^{3}$ Non-surgical medical and radiation-based oncological interventions are also associated with a risk of memory and other cognitive deterioration, including new neurological symptoms after adjuvant postsurgical radiation treatment. Currently, our incomplete understanding of the link between brain anatomy and function poses significant hurdles for developing individualised neuro-oncological treatment plans, especially in asymptomatic patients.

Long-range deep fibre bundles (figure 1) constitute the minority of WM connections but convey crucial information among functional systems. Putative behavioural contributions of individual fibre tracts were largely extrapolated from observations in neurological patients and animal experiments that underpinned seminal disconnection models. Our present-day understanding of the contributions of fibre pathways to complex behaviour has been further advanced by intraoperative electrical stimulation during neuro-oncological neurosurgery. ${ }^{3}$ With this growing appreciation of function, increasing efforts aim to evaluate how gliomas and their treatment affect fibre pathways.

Gliomas, especially high-grade gliomas, show features of infiltration on histological examination, including of 'secondary structures' such as WM fibres. These fibres, additionally, are often compressed, displaced or encapsulated by slow growing gliomas ${ }^{4}$ or may be destroyed by highgrade gliomas. Metastases may similarly compress or displace fibre tracts. With every patient, therefore, the physician is presented with a unique challenge to understand the relationship among tumour, structure and function in order to decide on treatment strategy. Consequently, a thorough knowledge of the functions mediated by fibre pathways is essential to minimise morbidity, while methods to evaluate fibre destruction or displacement in the individual patient occupy an increasingly prominent place in the neuro-oncological treatment of brain tumours.

\section{In vivo fibre tract identification}

The intricate WM architecture was initially uncovered through gross dissections, myelin staining and fixation-freezing techniques. Increasingly precise understanding of cortical connectivity has accumulated since the 1970s, with the emergence of anterograde and retrograde tracer techniques and recent developments in light microscopy. A fundamental drawback of these approaches is their reliance on invasive techniques.

The possibility to indirectly study fibre connections in the living human brain emerged with the development of MRI techniques sensitised to the random diffusion of water molecules and mathematical models to quantify directionality in water dispersion underlying diffusion tractography (DT).

The original and still widely pervasive DT analysis models divide into deterministic and probabilistic approaches. Deterministic algorithms trace, between one voxel and the next, the vectors representing the estimated direction of diffusion. This method produces beautiful renditions of fibre trajectories and is embedded within commercial neuronavigation systems. However, deterministic approaches offer no estimate of how likely a reconstructed 'tract' is. Probabilistic tractography 


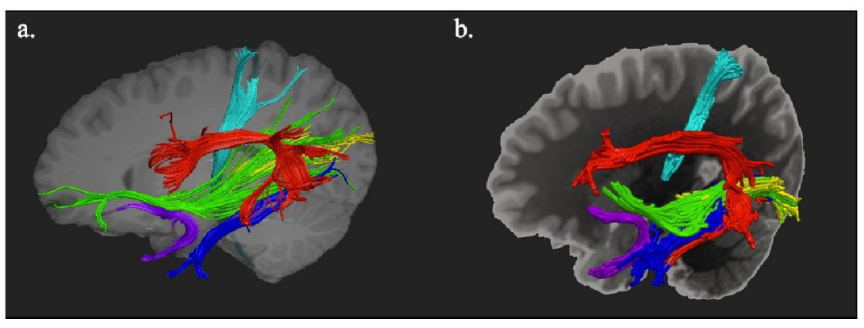

Figure 1 Major long association and selected projection fibre tracts in the human brain. Reconstruction of major white matter long-range association and selected projection fibre tracts using deterministic (A) and probabilistic (B) tractography algorithms. In the healthy brain, both methods produce similar tract courses in the deep white matter. While deterministic tractography-based reconstructions on clinical-grade diffusion data typically terminate at areas of low fractional anisotropy (FA), such as grey matter or perifocal oedema, probabilistic approaches are better able to track into such low FA areas where fibre orientations become less certain (see figure 2). Red, arcuate/superior longitudinal fasciculus, branch III; blue, inferior longitudinal fasciculus; green, inferior fronto-occipital fasciculus; purple, uncinate; yellow, optic radiations; cyan, corticospinal tract. This image is produced in colour online as part of the issue.

methods represent this uncertainty by 'sampling' connections passing through a voxel from a distribution of possible fibre orientations, to build statistical connection likelihood maps. ${ }^{5}$ Neither approach, when based on a single fibre-within-voxel model, can accurately capture complex WM organisation and instead may produce biologically non-existent (false positive) or erroneously omit (false negative) 'tracts'. This is because, for technical reasons, brain water diffusion is measured at the scale of cubic millimetres, far removed from the diameter $(\mu \mathrm{m})$ of fibres in the human brain. Consequently, multiple fibre populations often intersect in a diffusion-scale voxel.

We have previously outlined limitations and considerations for DT applications to neurosurgical planning. ${ }^{6}$ Evolving research algorithms that attempt to model microscale complexity offer vast improvements in DT sensitivity to cortical projections of tracts and in regions of crossing fibres (see Shi and Toga ${ }^{7}$ for a review). While these advanced techniques place significant demands on scanner hardware, acquisition time and postprocessing computational capacity, they are gaining increasing traction in presurgical settings due to improved delineation of crossing fibres (eg, Farquharson $\mathrm{et}_{\mathrm{al}} \mathrm{f}^{ }$) and reduced sensitivity to partial volume effects in areas affected by peritumoural oedema. ${ }^{9}$

\section{Linking connectivity with function}

Notwithstanding methodological considerations, the non-invasive nature of diffusion MRI and lack of other methods to assess the course of fibre tracts in vivo have driven an increased uptake of tractography techniques in neurosurgical oncology settings as a means to inform both the likelihood of achieving a complete resection and risks of inducing functional deficits. In this context, it is important to consider that DT does not directly measure anatomy and is sensitive to pathological features such as oedema (figure 2), which can reduce the certainty of directional estimates and, thus, tractography results. ${ }^{6}$ Crucially, however, even knowing the precise location of a fibre tract does not inform the function of those fibres. Such limitations have led to controversy surrounding the neurosurgical utility of DT, when compared with direct electrical stimulation (DES). Yet, although intuitive, direct comparisons between DT and DES are

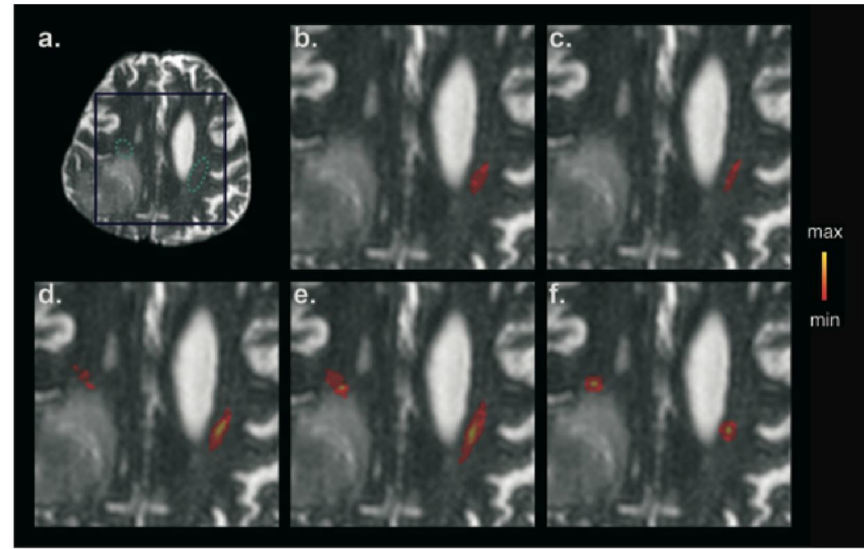

Figure 2 Impact of tractography analysis choice on reconstruction of the corticospinal tract. Case illustration in a right retro-Rolandic glioblastoma with contralateral hemiparesis surrounded by perifocal oedema on nondiffusion T2-weighted EPI. (A) Representative section: areas, through which fibres of the pyramidal tract pass, are circled in green dotted lines. (B-F) Performance of five different tracking algorithms: simple (B) and interpolated streamlining (C), probabilistic tractography without (D) and with crossing-fibres modelling $(\mathrm{E})$ and according to the constrained Bayesian framework (F). While overall correspondence is reasonable in successful reconstructions, spatial tract latitudes differ, and streamlining algorithms were false negative on the lesion side (red to yellow: above-zero number of samples reaching target from seed scaled between minimum (red) and maximum (yellow), red only for minimum equals maximum of deterministic streamlining). This image is produced in colour online as part of the issue.

complicated by differences in what is being measured by each technique and why.

DT currently offers the only technique to link the unique connectional organisation of an individual's brain with (separate) measures of how that person processes information. Although multiple tools have been developed to estimate or modulate cortical activity, measuring information processing in the brain, especially through WM, remains a phenomenal challenge. In neuro-oncological settings, efforts have focused on DES applied to WM to transiently disrupt (or elicit abnormal) function as a means to establish causality and predict the consequences of surgical resection in a specific location. ${ }^{3}$ While DES offers a powerful probe of behaviour in the area of surgically exposed tissue, its results depend on stimulation thresholds, the specific (time limited) tests administered and fluctuations in patients' cooperativeness. Crucially, it is unknown whether fibre bundles are functionally uniform along their length. For example, DES more often produces behavioural errors when applied to the posterior than to the anterior inferior longitudinal fasciculus. ${ }^{10}$ Major challenges arise in precisely localising DES effects to individual pathways, especially where multiple tracts converge and may jointly contribute to functions, or in areas of complex fibre arrangement. In such regions, the orientation of stimulation relative to fibres must be inferred, since axon bundles cannot be distinguished visually and may be displaced by tumour mass effects or brain shift during debulking. Thus, while DES—when feasible-is invaluable to evaluate and limit functional risks during resection, it provides alternative information to DT and faces different challenges in inferring connectional anatomy.

No perfect technique exists to measure fibre pathways and their functions in the living human brain. Yet, better knowledge of the impact of focal neuro-oncological treatment at the brain systems 
level speaks to the quest for personalised treatment planning in patients who are increasingly surviving longer with brain tumours. Numerous excellent reviews have summarised the known longrange connectional anatomy of the human brain. Our objective is to extend this literature by supplementing evidence that damage to select fibre pathways bears directly on behavioural outcomes in the context of neuro-oncological treatment. While the mainstay of DT applications presently is in surgical oncology, we propose that DT may also inform and potentially help reduce radiation-induced memory and cognitive impairment by planning radiation fields and drop-off on diffusion MRI. Our hope is to encourage greater discussion surrounding the impact of fibre integrity and preservation in the context of surgery and along the complete therapeutic pathway from early diagnosis through to oncological therapy, starting from a deeper understanding of the anatomy and functional role of WM.

\section{Functional considerations for treatment}

While some risks associated with neuro-oncological interventions are invariant, the specific location of a tumour can dictate, with increasing precision, the functional circuits that stand to be disrupted by treatment. Functional risks are prominent in the frontal lobe, given the involvement of its long-range fibres in movement and speech. However, recent interest has also turned to attention and memory, being among the greatest concerns of adult brain tumour patients. ${ }^{11}$

\section{Corticospinal tract and movement}

In terms of motor function, deep brain tumours that do not involve precentral cortex may nonetheless compromise motor functions when pathological processes infiltrate or exert pressure on corticospinal tract (CST) fibres. Accordingly, intraoperative DES of CST fibres during glioma resection typically elicit involuntary motor responses or disrupt precise sensorimotor function. ${ }^{3}$

Since infarcts or accidental surgical damage can result in debilitating motor impairment, visualising the course of CST fibres (table 1) in relation to an intracranial tumour was among the first highlighted potentials of DT. Although consistent in the medial WM containing the trunk of CST fibres, ${ }^{12}$ standard DT reconstructions are highly variable in lateral cortical projections, mirroring substantial individual differences myelo-architectonically $^{13}$ (and reflecting methodological variations in tractography algorithms). Probabilistic tractography based on diffusion data with high angular resolution improves visualisation of laterally projecting CST fibres ${ }^{14}$ compared with deterministic algorithms. Reconstruction of cortically directed CST projections may be further enhanced using complementary functional measures derived from functional MRI or navigated transcranial magnetic stimulation during analysis. ${ }^{15}$ Consequently, CST tractography is advocated as clinically helpful to determine when resection should be continuously alternated with subcortical DES to maximise oncological benefit while preserving critical motor function. ${ }^{414}$ In our experience, DT may be especially helpful when tumours are located in deep cortical structures or within/just below the central sulcus, in order to help identify whether CST fibres are located anterior, posterior or within the lesion (figure 3).

The direct impact of CST tractography on surgical practice has, however, only rarely been evaluated through randomised trials. In a large group of neurosurgically treated patients, significantly fewer motor deficits (and increased life expectancy in high-grade subgroups) were noted in cases where DT was fused with neuronavigation, compared with neuronavigation alone. ${ }^{17}$ Morbidity rates should improve further by including in such studies subcortical DES and/or intraoperative neurophysiology to maximise safe resections close to the CST.

\section{Speech: superior longitudinal fasciculus (SLF) and arcuate}

Intersecting with CST fibres at the level of the centrum semiovale are frontal lobe fibres relevant for speech. In the classical model of aphasia, disorders of language could arise from damage to Broca's (inferior frontal) and Wernicke's (posterior superior temporal) areas or to a pathway between them. Von Monakow is credited with suggesting that the arcuate fascicle provided such a candidate pathway. However, modern non-human primate tract tracer studies challenged the existence of direct connections between these cortical regions via the arcuate fasciculus and, instead, identified a system of multiple-at least partially separate-long-range frontal lobe fibre bundles (exquisitely described by Schmahmann and Pandya ${ }^{18}$ ). In humans, debate continues around the exact anterior cortical terminations of the arcuate, with the balance of evidence indicating predominant connections to pars opercularis. The connectional complexity of SLF and arcuate fibres (table 1) drive uncertainty concerning their functional specialisation. However, it remains undisputed that some of these fibres make essential contributions to language. Accordingly, from its earliest days, DT was employed to plan surgical approaches to gliomas in proximity to these pathways.

The complex SLF/arcuate system was visualised with DT over 10 years ago ${ }^{19}$ and has been corroborated in multiple DT-dissection comparison studies. Arcuate (or 'SLF-IV') DT reconstructions show high variability in their cortical endpoints but closely match anatomical dissections in their subcortical course. ${ }^{20}$

DES along the arcuate in the dominant hemisphere routinely disrupts speech production, inducing phonemic paraphasias and sometimes repetitions. ${ }^{3}$ In contrast, DES applied near the lateral SLF-III fibres produces dysarthria or anarthria, ${ }^{3}$ supporting contributions to motor articulatory aspects of speech. After resections up to these tracts, patients tend to exhibit transient speech disorders, ${ }^{21}$ while infarcts affecting SLF/arcuate fibres disrupt phonological processing ${ }^{22}$ and reduce speech fluency. ${ }^{23}$ These clinical data suggest that, in humans, the dominant-hemisphere arcuate and SLF-III may constitute a 'dorsal phonological route' of speech monitoring, planning and execution in modern parallel stream models of language. ${ }^{24}$

The primary clinical challenge has remained how to evaluate the clinical impact of arcuate/SLF-III DT to improve surgical outcomes. An elegant recent study evaluated longitudinal preoperative and postoperative DT against postsurgical language outcomes. All patients with preserved arcuate and temporo-parietal fibres showed preserved language following surgery, whereas damage to either pathway predicted persistent deficits 6 months postsurgery. ${ }^{21}$ Similar findings have been reported in smaller case studies and highlight an additional postoperative indication of DT to prognosticate recovery of early postoperative deficits. ${ }^{21}$

\section{Frontal aslant fibres}

For both movement and speech, connections of the supplementary motor area have received interest due to the characteristic 'supplementary motor area (SMA) syndrome' resulting from neurosurgical resections in this area. A frontal aslant tract, linking the precentral cortex and pars opercularis with the SMA, has been identified in humans using DT and dissections ${ }^{25}$ and ascribed a role in speech initiation and orofacial movement control. SMA syndromes raise fascinating questions about 
Neurosurgery

Table 1 Connectional anatomy and neuro-oncological relevance of white matter fibre tracts

\begin{tabular}{|c|c|}
\hline Fibre tract & Anatomy \\
\hline Corticospinal tract (CST) & $\begin{array}{l}\text { The pyramidal tract is formed by corticospinal fibres, which control movements of the contralateral } \\
\text { limbs and trunk, and corticobulbar fibres, which innervate the cranial nerves controlling face, neck and } \\
\text { head movements. Pyramidal tract fibres arise predominantly from neurons in the upper precentral gyrus } \\
\text { with important supplemental inputs from other frontal, parietal and insular neurons. From upper motor } \\
\text { neurons, axons that form the CST descend through the posterior limb of the internal capsule to the cerebral } \\
\text { peduncles and into the spinal cord. Despite interindividual variability in the volume of CST fibres and their } \\
\text { exact location, within the internal capsule, CST fibres generally follow a progressively more caudal route } \\
\text { through the posterior limb of the internal capsule as they descend. Between the cortex and the internal } \\
\text { capsule, the CST constitutes a subpart of the fanning fibres forming the corona radiata. The orientation of } \\
\text { the somatotopically organised CST fibres changes from a left-right distribution in the cortex (corresponding } \\
\text { to the motor homunculus) to anterior-posterior through the internal capsule and peduncle. Cranial nerve } \\
\text { nuclei } V, I X, X \text { and upper VII receive bilateral innervation, while other nuclei are innervated unilaterally. }\end{array}$ \\
\hline Arcuate fasciculus & $\begin{array}{l}\text { In monkeys, links the superior temporal gyrus with the dorsolateral prefrontal and premotor cortices, as wel } \\
\text { as the homologue of pars opercularis but only rarely with pars triangularis. } \\
\text { In humans, anteriorly, the arcuate fibres in the white matter above the sulcus semicircularis insulae } \\
\text { converge with those of the lateral SLF and inferior fronto-occipital fasciculus (IFOF) which are difficult to } \\
\text { differentiate in blunt dissections. }\end{array}$ \\
\hline $\begin{array}{l}\text { Superior longitudinal } \\
\text { fasciculus, branch III (SLF-III) }\end{array}$ & $\begin{array}{l}\text { Located at the level of the frontal and parietal operculum. Connects pars opercularis and inferior aspects of } \\
\text { premotor and dorsolateral cortex to the homologue of human supramarginal gyrus in monkeys. }\end{array}$ \\
\hline IFOF (or IOFF) & $\begin{array}{l}\text { Subcortically, IFOF fibres occupy the white matter of the frontal operculum (either side of the claustrum) } \\
\text { and course inferiorly through the dorsal temporal stem where they merge gradually with the uncinate } \\
\text { fasciculus below it. The IFOF continues posteriorly above the roof of the temporal horn, then aligns parallel } \\
\text { and lateral to the optic radiations, remaining medial to those of the ILF and arcuate next to the atrium of } \\
\text { the lateral ventricle. Its cortical terminations remain elusive and, on myelin staining, appear highly variable. } \\
\text { Anterior fibres have been tracked mediolaterally into pars triangularis and orbitalis and superiorly into } \\
\text { dorsolateral prefrontal white matter. Posteriorly, connections are reported to all occipital gyri and, less } \\
\text { frequently, the fusiform and parietal cortices. }\end{array}$ \\
\hline
\end{tabular}

Frontal aslant tract $\quad$ Connects the precentral cortex and pars opercularis with the supplementary motor area. Frontal aslant fibres appear to terminate at the border between the supplementary motor area (SMA) and SMA proper.

Clinical impact of damage

Pareses

Phonemic paraphasias, repetitions, non-fluent 'expressive' aphasia

Dysarthria, anarthria

(Visual) semantic deficits during naming

Potentially aspects of emotional recognition/ processing

SMA syndrome (variable, mostly transient loss in movement and speech function)

Inferior longitudinal fasciculus Temporopolar ILF fibres are positioned at the level of the inferior temporal gyrus and run posteriorly to (ILF) the superior and middle occipital gyri, remaining lateral to the optic radiations but medial to the SLF. Some medial connections are traced to the fusiform and posterior inferior occipital gyrus. As with other association fibres, the ILF has a relatively invariant core, with greater intersubject variability in cortical terminations.

Middle longitudinal fasciculus In monkey, fibres along the length of the superior temporal gyrus link the inferior parietal lobe with the (MdLF) (superior) temporal pole, running parallel but laterally to the extreme capsule fibres until these tracts separate at the level of the posterior insula. In human, diffusion tractography (DT)-derived cortical terminations in the angular gyrus have been questioned and instead observed further posteriorly in the occipital lobe in dissections.

Temporo-parietal branch of the SLF (SLF-tp)

Connects temporal (posterior inferior, middle and some superior temporal gyrus) with parietal (angular and supramarginal) cortex. Some connections have also been noted in the inferior middle occipital gyrus.

Visual agnosias, including pure alexia (posterior fibres) and potentially prosopagnosia

Not established

In the language-dominant hemisphere: aphasia (most likely fluent or 'receptive')

\begin{tabular}{lll}
\hline SLF-I & Runs dorsal to the cingulate sulcus and connects the medial and caudal parietal lobe with the SMA and & Not established \\
premotor, superior frontal and dorsomedial frontal cortex. Recently described in detailed human dissections. & Neglect syndromes \\
SLF-II & Connects the angular gyrus with premotor and dorsolateral middle and superior frontal regions. Recently \\
& described in detailed human dissections. & Visual field defects \\
Optic radiations & Formed by three bundles connecting the lateral geniculate nucleus (LGN) with the occipital pole. & (quandrantanopia, hemianopia) \\
& Ventral projections run from the LGN toward the anterior tip of the temporal horn, where they turn \\
& backwards, forming the 'Flechsig-Meyer(-Cushing-v.Archamblaut)' loop, and continue parallel to the \\
& atrium of the lateral ventricle into the lower bank of the calcarine fissure. \\
& Dorsal optic radiations curve from the LGN along the occipital horn into the upper calcarine bank. \\
& The central bundle courses lateral to the roof of the temporal horn and turns backwards along the \\
& occipital horn, above and parallel to the ILF, into the occipital pole. & \\
\hline
\end{tabular}

dynamic connectivity, given that symptoms generally improve with time. DT of these fibres at present is predominantly informative when preoperatively counselling patients and their families of usually transient but potentially prolonged debilitating symptoms.

\section{Semantic knowledge and the inferior fronto-occipital fasciculus (IFOF/IOFF)}

Normal language processing involves a complex series of computations including the recognition, communication and association of meaning. Observations of reproducible and specifically semantic language errors during subcortical stimulation in glioma surgery ${ }^{26}$ identified the IFOF/IOFF as a plausible anatomical substrate for a likely bilaterally represented 'ventral semantic' stream. ${ }^{24}$ The existence of such a tract has long been shrouded in controversy, since it may be unique to humans. ${ }^{27}$

Described in human dissections using the reinvigorated Klingler technique, the IFOF is situated in the WM either side of the claustrum, coursing through the dorsal temporal stem and continuing in between the optic radiations and the ILF on into 


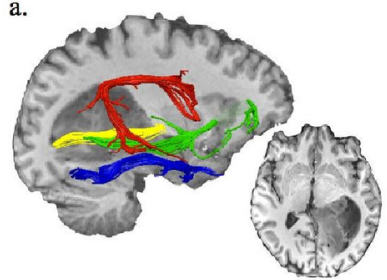

c.
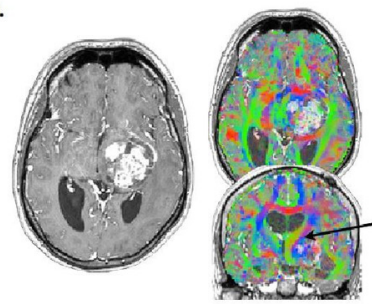

b.

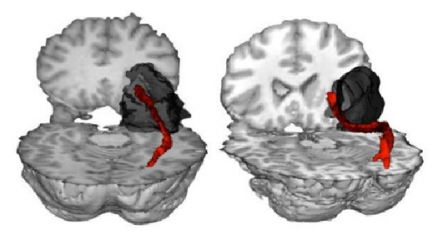

d.

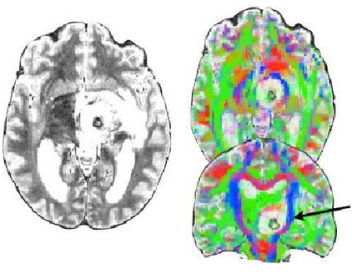

Figure 3 Diffusion tractography in neuro-oncological settings. Clinical cases illustrating select applications of white matter fibre imaging in neurosurgical oncology decision making. (A) To access this glioma in the dominant hemisphere, the most direct lateral surgical approach would risk transecting functionally eloquent pathways. Tractographic reconstructions of the arcuate (red), optic radiations (yellow) inferior longitudinal fasciculus (blue) and inferior fronto-occipital fasciculus (green) identified a posterolateral access window avoiding all major fibre bundles. Phonemic paraphasias, wording finding difficulties, the inability to recognise the meaning of presented pictures and visual disturbance in the contralateral visual field were induced by subcortical stimulation at the location of reconstructed fibres and defined the maximal resection boundaries. (B) Tractography reconstructions of the inferior fronto-occipital fasciculus (red) in two representative patients illustrating common encasement (left) and displacement (right) of this tract by a low-grade glioma (rendered as a 3-dimensional mask in grey). These preoperative reconstructions assist in planning surgical stages and the optimal timing of intraoperative awake stimulation. (C) Two illustrative cases in which white matter imaging informed the displacement of corticospinal tract fibres (black arrows) relevant for biopsy planning. In the first case, direction-coded maps (in which red denotes preferential diffusion in the left-right/right-left direction, green in the anterior-posterior/posterior-anterior direction and blue in the inferior-superior/superior-inferior direction) revealed displacement of corticospinal fibres medioanteriorly, permitting access for biopsy and complete resection from a lateral approach with full preservation of postoperative motor function. Conversely, in the second case, the same lateral access route would directly transect the laterally displaced corticospinal tract fibres. This image is produced in colour online as part of the issue.

the occipital lobe (table 1). DT studies reveal a tract that matches this course with high consistency. ${ }^{27}$

Preoperative DT mapping is particularly relevant due to frequent infiltration (approximately 33\%) or displacement (approximately $40 \%$ ) of IFOF fibres by gliomas ${ }^{4}$ (figure 2). The concordance between DES-related semantic errors and IFOF DT fibre reconstructions is generally high (above $80 \%$ ). ${ }^{28}$ Importantly, failure to reconstruct the IFOF with DT, when attributable to apparent infiltration, reportedly carries a high positive predictive value $(87.5 \%-$ $100 \%)$ that no tracts will be found with DES. ${ }^{4}$ However, in terms of permanent deficits, because DES-elicited errors constitute the stopping point for oncological resections, any postoperative language deficits are typically transient and difficult to attribute uniquely to IFOF damage. Yet recently, a preliminary voxel-based lesion mapping study in stroke patients found semantic naming deficits to associate with IFOF damage. ${ }^{29}$ Neurological studies

suggest that IFOF fibres may additionally contribute to aspects of face processing, although evidence of selective face recognition deficits associated with oncological damage is currently lacking.

Visual recognition: inferior longitudinal fasciculus (ILF)

While neuroimaging and lesion studies indicate essential contributions of temporal cortex to aspects of language, remarkably little is known of the behavioural role of long-range temporal lobe fibres. Extensive U-fibres and complex recurrent projections contribute to ongoing uncertainty surrounding specialised functions mediated by fibres in the occipitotemporal system

DT descriptions replicate fibre dissection studies showing a single long association tract-the ILF-in the deep inferior temporal lobe WM linking the temporal pole with the superior, middle and inferior temporal gyri ${ }^{30}$ (table 1$)$. Case reports have related selective reading impairments (ie, pure alexia without agraphia or hemianopsia) to disruptions of dominant hemisphere $\operatorname{ILF}^{31}$ and vertical occipital ${ }^{32}$ fibres connecting the 'visual word form area' near the occipitotemporal sulcus to the occipital cortex and the inferior parietal lobe, respectively. Additionally, impaired face recognition (prosopagnosia) has been ascribed to WM disruption near the fusiform gyrus encompassing ILF fibres.

On standard picture naming paradigms, intraoperative DES of the (presumed) ILF elicits picture-naming deficits (anomia) only variably and sometimes not at all. ${ }^{10}$ These findings underpin neurosurgical perspectives that some anterior ILF fibres can be resected without long-term consequences for language. ${ }^{10}$ Instead, using a battery of test stimuli in three patients, DES-induced disruption during reading and common symbol recognition supports contributions of posterior ILF fibres to visual recognition. ${ }^{33}$ These findings highlight the crucial importance of task selection in the detection of deficits during neuro-oncological surgery. Indeed, the evolution of cognitive deficits following neuro-oncological resections has only recently become the topic of active research, which may uncover important contributions of anterior ILF fibres to, for example, emotional learning.

\section{Middle longitudinal fasciculus (MdLF)}

An additional temporal lobe fibre pathway has been described in the superior temporal WM using DT, although less frequently in human dissections $^{34}$ (table 1). This MdLF is considered among the 'ventral' language pathways, whose unique neuro-oncological relevance remains to be refined. Potential contributions to comprehension and language perception have been proposed. Yet, surgical stimulation and resection of anterior and middle MdLF portions in eight patients induced neither naming deficits intraoperatively nor new language deficits postoperatively. ${ }^{35}$ Instead, the authors suggest the MdLF could contribute to untested functions such as multimodal integrative functions involving the angular gyrus.

\section{Language: the temporo-parietal branch of the SLF (SLF-tp)}

A final set of language-relevant connections is proposed to link the temporal lobe with the parietal cortex. 'Tracts' linking the middle and posterior temporal cortex with the inferior parietal cortex (table 1) are consistently observed in DT. Originally called the 'vertical branch' of the arcuate, these fibres are positioned lateral to the arcuate,${ }^{34}$ leading to the alternative nomenclature of 'SLF-tp'. Although not always detected, ${ }^{34}$ a fibre bundle following the course described by DT has been observed in human brain dissections. ${ }^{20}$

Due to proximity of multiple critical WM pathways, including the anterior portions of Wernicke's vertical occipital fasciculus, 
it is challenging to ascribe intraoperative DES phonological and repetition errors uniquely to the SLF-tp. However, retrospective studies identified damage to connections linking the superior temporal and angular gyri as predictive of aphasia after stroke ${ }^{36}$ and found surgical damage to the SLF-tp or arcuate to result in sustained language deficits. ${ }^{21}$

\section{Attention and the SLF-II}

In contrast to language functions mediated by inferior parietal connections, mounting evidence from poststroke neglect suggests fibres interlinking the dorsal parietal and frontal lobes, in particular SLF-II (table 1), contribute to visuo-spatial attention. Although neglect syndromes arise from damage to pathways other than the SLF, during awake glioma surgery, errors during basic line bisection have been observed only during DES in locations consistent with SLF-II. ${ }^{37}$ Yet, at present, deficits arising from neuro-oncological damage or resection specific to SLF-II and neighbouring SLF-I fibres remain ill defined.

\section{Vision and the optic radiations}

Finally, treatment of tumours located in the occipital lobe may disrupt basic visual processing. Visual field defects may arise from damage to any part of the optic radiations, which are formed by three bundles (ventral, dorsal and central) carrying the projections of the upper and lower visual fields and macular fibres, respectively ${ }^{38}$ (table 1). The ventral projections raise the greatest surgical concerns to avoid visual field deficits after anterior temporal approaches. However, superiorly, optic radiations extend up to the level of the superior temporal sulcus and are, therefore, relevant when planning posterior lateral surgical approaches in the temporal lobe as well.

DT-based reconstructions show high concordance with ex vivo delineations of all three optic bundles ${ }^{39}$ and mirror variability reported in both myelin staining studies ${ }^{40}$ and fibre dissections, especially in anterior extents. ${ }^{38}$

Optic fibres have been monitored intraoperatively during awake glioma neurosurgery but rarely in combination with DT, although correlations have been reported between visual-evoked potentials and DT-derived optic radiation locations. ${ }^{41}$ Instead, based on the high fidelity of optic radiation DT, numerous studies have investigated the utility of DT to predict visual loss after neurosurgical interventions. A recent meta-analysis of 13 studies concluded that DT results accurately predict surgical damage to the optic radiations in individual patients, ${ }^{42}$ which can be specifically avoided though optic-radiation navigated neurosurgery conducted under general anaesthesia. ${ }^{43}$

\section{Impact on neuro-oncological decision making}

Since the earliest applications 15 years ago, accumulating data highlight select applications for DT foremost in planning and performing first-line neuro-oncological surgery. From a neurological perspective, understanding how a patient's symptoms relate to the involvement of distinct fibre bundles informs both the likely cause for presenting symptoms and relative potential for recovery following successful treatment. The frequent displacement and infiltration of fibre bundles have driven a particular uptake of DT in planning awake surgeries for low-grade gliomas. Adopting the philosophy of maximal resections for optimised oncological benefit, ${ }^{2}$ an estimate, provided through DT, of the proximity or involvement of specific fibre tracts in the deep WM helps to anticipate the likely resection volume achievable and facilitates discussions with patients about the expected nature of postoperative deficits, should they wish to pursue surgery (figure 3 ). If the surgical access or intended resection extent indicates awake surgery to minimise functional risks, transfer of DT results into neuronavigation can be beneficial to focus DES, ${ }^{14}$ provided that geometric image distortions of diffusion-weighted imaging are adequately corrected, and brain shift is carefully considered (eg, using navigated ultrasonography). In this way, DT can help to minimise awake stimulation times, patient discomfort and seizure risks. ${ }^{4}$ In our hands, DT-navigated, DES-guided awake surgery has also reduced the incidence and severity of postoperative deficits and, accordingly, the length of postoperative hospital stays. ${ }^{44}$

New prospects for DT applications are arising in emerging neuro-oncological surgical arenas. MRI-guided stereotactic laser interstitial thermal therapy has been evaluated for potential efficacy to ablate small residual or recurring metastatic and primary brain tumours and palliative treatment of newly diagnosed highgrade tumours not amenable to resection. Following laser ablation, new postoperative deficits, predominantly transient and motor-related in nature, have been noted in 16\%-20\% of such patients and retrospectively attributed to postoperative oedema or 'thermal injury' affecting the corticospinal tract. ${ }^{45}$ The utility of DT to monitor and prevent accidental insertion- or thermal-related damage to key fibre tracts has been proposed in individual cases, ${ }^{46}$ but not yet systematically investigated. A further potential indication is in planning access routes for minimally invasive endoscopic surgery, although DT-randomised trials are presently lacking.

As modern neuro-oncological treatments are prolonging life expectancy, an increasing emphasis is placed on optimising the quality of years gained by focused intervention, including radiotherapy. In particular, there has been a growing focus on understanding and minimising cognitive declines observed after radiation therapy. The mechanisms for radiation-induced cognitive impairments remain obscure. In addition to disrupted neurogenesis and chronic inflammatory responses, ${ }^{47}$ radiotherapy is associated with a greater incidence of WM hyperintensities and may induce subtle progressive WM disturbance. ${ }^{48}$ Crucially, careful surgical preservation of tracts may theoretically be undone if radiotherapy compromises important proximal tracts. Longitudinal DT measurements suggest that greater reductions in fibre tract integrity, reflecting dose-dependent effects of radiation therapy, ${ }^{49}$ predict greater cognitive declines following treatment. ${ }^{50}$ An intriguing idea, therefore, is to use DT information to minimise radiation doses delivered to portions of fibre tracts with high sensitivity to radiation effects. ${ }^{51}$ Integrated DT was used to compare radiation doses delivered to the hippocampus and lesion-adjacent fibre tracts during proton and photon-based radiotherapies in high-grade glioma patients. ${ }^{52}$ Inclusion of tractography results reportedly enabled preservation of functionally relevant fibre pathways during CyberKnife treatment planning for metastases, tumours and arteriovenous malformations ${ }^{53}$ and significantly reduced functional complications following stereotactic radiosurgery in the latter group. ${ }^{54}$ The functional benefits of fibre-centric radiation treatment plans clearly will need assessment through randomisation studies in larger series focusing on specific brain neoplasms. Yet, DT holds particular future promise to develop individualised treatment approaches aimed at monitoring-and potentially preventingdosimetry-related disruption to core fibre tracts.

Although not directly dependent on tractography, measures of WM microstructure derived from directionally encoded diffusion-weighted images deserve mention, as these offer promising avenues for tumour differentiation or staging as well as treatment response monitoring (reviewed in $\mathrm{Li}$ and Padhani ${ }^{55}$ ). Quantitative 
diffusion indices may similarly contribute insight into potential cognitive and neurological consequences of non-targeted radiotherapy and chemotherapy. Cognitive side effects in subgroups of chemotherapy survivors likely have multifactorial origins, including shared risk factors for cancer and cognitive decline as well as DNA damage, disrupted repair mechanisms and accelerated ageing. ${ }^{56}$ Longitudinal diffusion MRI studies in non-CNS cancers have identified widespread changes in WM microstructure suggestive of demyelination and axonal damage pre-topost chemotherapy. ${ }^{57}$ Interestingly, these WM changes tend to normalise within a few (3-5) years following treatment. ${ }^{58} \mathrm{~A}$ direct role for DT is not currently proposed for guiding systemic treatments. However, monitoring acute WM disruption in relation to specific chemotherapeutic agents and emerging delivery approaches (such as convection-enhanced delivery) may prove useful to assay toxicity, predict cognitive deficits and/or assess eventual neuroprotective interventions. We do not here discuss the great interest surrounding the utility of quantitative (rather than tractographic) diffusion MRI metrics to predict radiotherapy and/or chemotherapy treatment response as this topic has recently been comprehensively reviewed. ${ }^{59}$

\section{Brain networks and recovery}

Emerging concepts in brain organisation increasingly emphasise the role of each person's unique connectional architecture on neuronal function. Despite an increasing focus on brain network preservation, no clinical tools currently exist to evaluate wholebrain organisation.

The application of DT (in combination with functional MRI measures of neuronal activity) to neuro-oncology and neurological conditions offers unique opportunities to refine our appreciation of human network information processing and its disruption through tumour growth and treatment. One potentially important impact of network-based analysis models is in monitoring an individual brain's adaptive potential to optimally time multistage or adjuvant neuro-oncological therapies. Preliminary reports suggest that cortical functional limits, encountered with DES during surgery for low-grade gliomas, may redistribute over time. ${ }^{60}$ These remarkable findings raise crucial questions. How does such functional reorganisation in the cortex relate to relatively more rigid $^{1}$ structural brain connectivity? What amount of damage can be tolerated by a 'tract' before deficits become permanent? What are the characteristics of individual tumours that promote or limit neuroplasticity processes from occurring? What are the relevant timelines for behaviourally efficient plasticity, and how are these affected by different neuro-oncological treatments?

The ready availability of MRI technology and the potential to safely acquire longitudinal measurements (figure 4) provide opportunities for DT to answer these unknowns and assess and track the impact of neuro-oncological treatments on the circuitry underlying functional brain networks over time. In this way, DT may help to uncover structural substrates underlying individual differences in rehabilitative potential following neuro-oncological interventions, for example, variability in hemispheric tract symmetry, as has been proposed poststroke. ${ }^{61}$ While intuitively, slow-growing low-grade gliomas may gradually affect information transfer along fibre tracts in a manner conducive to adaptive reorganisation across functional networks, similar processes could possibly also occur in secondary high-grade gliomas harbouring prognostically beneficial isocitrate dehydrogenase (IDH) mutations. A major challenge is to demonstrate the longterm efficiency and potential limits of such plasticity as well
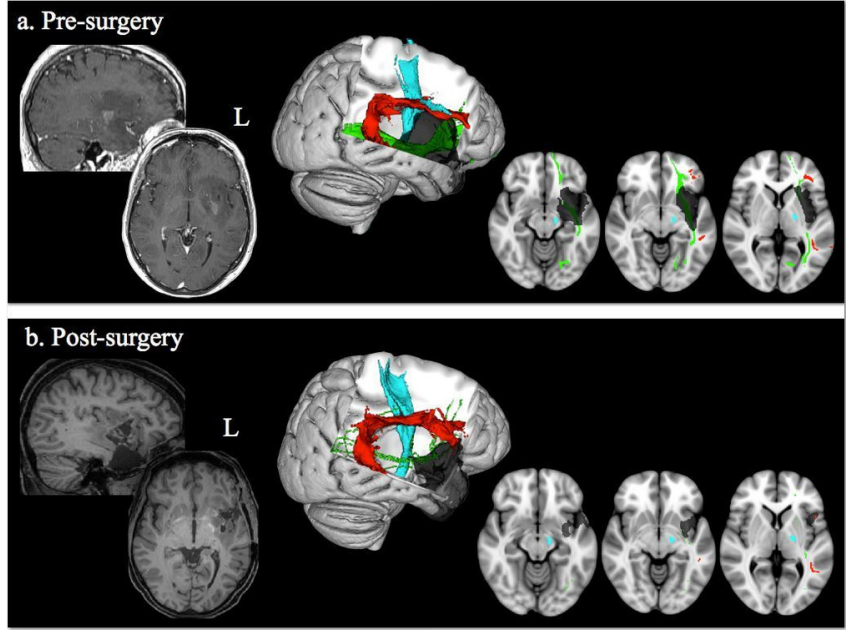

Figure 4 Longitudinal tractography visualisation of fibre disruption. Clinical case illustrating longitudinal diffusion tractography applications to evaluate fibre disruption in the context of oncological intervention. Preoperative (A) reconstruction of the inferior frontooccipital fasciculus (IFOF) (green), corticospinal tract (cyan) and arcuate/ superior longitudinal fasciculus, branch III (red), identified risks of resecting a temporo-insular glioma (black) to the tumour-encased IFOF fibres, as well as to arcuate fibres approaching the anterior tumour margin. Intraoperatively, persistent semantic errors were encountered on stimulating the temporal stem white matter at the location of the IFOF. Fluctuating speech disorder and phonemic paraphasias marked the anterior resection boundary in the insula and inferior frontal cortex. Repeat tractography conducted 1 month after surgery (B) showed a reduction in IFOF fibres at the medial extent of the resection cavity (black). Postoperative neuropsychology revealed intact repetition, auditory naming and fluency, but persistent impaired picture naming.

as shared versus diverging principles across neuro-oncological lesions. It has been suggested that, for certain functions, 'indirect' functional routes may be able to mediate information processes previously supported by surgically damaged pathways. ${ }^{10}$ An alternate possibility is that differences in behavioural outcomes following fibre tract damage do not reflect plasticity per se, but instead reveal differences in behavioural strategies, such as favouring phonological or semantic processing during reading. Finally, tract degeneration secondary to surgical damage is a much-overlooked consideration that may nonetheless contribute significantly to behavioural deficits. The potential to gain understanding, through DT studies, of the incidence and behavioural ramifications of such processes following glioma surgery holds promise in the future to personalise and maximise therapies according to quantifiable measures of individuals' rehabilitative potential and progress. Whether substantial and beneficial behavioural adaptation can be promoted following surgery or targeted radiotherapy and remains confined to the special case of gliomas offers exciting avenues for further research.

\section{CONCLUSION}

Since seminal descriptions of the brain's intricate connectional architecture at the turn of the 19th century, neurological, neurosurgical and neuroscientific observations have dramatically improved our knowledge of the essential functional contributions of WM fibre pathways. Although not a direct measure of anatomy, DT - as the sole method to study fibre orientations in the living human brain-has proven a valuable tool to augment 
our understanding of connectional circuits supporting brain function.

As increasingly sophisticated diffusion acquisition and modelling approaches emerge and are adopted by the neuro-oncology community, it becomes ever more important to evolve clinical neuroanatomical training, develop consensus protocols for clinical DT acquisition and analysis and standardise evaluations of tractography's impact on the outcomes achieved with various oncological treatments. For example, the majority of DT evaluations are based on minimal (six direction) data, analysed with deterministic approaches, an approach that is particularly susceptible to fail in areas of oedema or crossing fibres (figure 2). Instead, studies comparing basic and advanced algorithms highlight that probabilistic tractography and multifibre models provide results that better represent anatomical complexity in cortical terminations of fibre tracts ${ }^{14}$ and better predict surgical outcomes ${ }^{21}$ than deterministic DT results. High variability in acquired data quality, as well as user-defined analysis approaches, fundamentally limits evaluations of DT's clinical utility. This hindrance is recognised in well-established neuro-oncology surgery applications and will likely impede translation to newly emerging applications in laser therapy and radiotherapy planning, unless a minimal set of standardised methods are adopted across DT users.

Ultimately, the clinical impact of DT in neuro-oncological settings depends on its contribution to maximising and sustaining a meaningful quality of life. Even more than DT or DES methods, standardisation in the preoperative, intraoperative and postoperative cognitive and neurological assessment of glioma patients will become increasingly crucial to isolate anatomical predictors of specific functional declines and the optimally sensitive methods to preoperatively detect and preserve these.

Contributors All authors contributed to literature searches, interpretation and drafting of the manuscript.

\section{Competing interests None declared.}

Provenance and peer review Not commissioned; externally peer reviewed.

(c) Article author(s) (or their employer(s) unless otherwise stated in the text of the article) 2017. All rights reserved. No commercial use is permitted unless otherwise expressly granted.

\section{REFERENCES}

1 Herbet G, Maheu M, Costi E, et al. Mapping neuroplastic potential in brain-damaged patients. Brain 2016;139:829-44.

2 Hervey-Jumper SL, Berger MS. Technical nuances of awake brain tumor surgery and the role of maximum safe resection. 2015:59:351-60.

3 Duffau $\mathrm{H}$. Stimulation mapping of white matter tracts to study brain functional connectivity. Nat Rev Neurol 2015;11:255-65.

4 Bello L, Castellano A, Fava E, et al. Intraoperative use of diffusion tensor imaging fiber tractography and subcortical mapping for resection of gliomas: technical considerations. Neurosurg Focus 2010;28:E6.

5 Jones DK. Studying connections in the living human brain with diffusion MRI. Cortex 2008;44:936-52.

6 Bartsch AJ, Biller A, Homola GA. Presurgical tractography applications. In: JohansenBerg H, Behrens TE, eds. Diffusion MRI: From Quantitative Measurement to InVivoNeuroanatomy, 2014. 2 ed.

7 Shi Y, Toga AW. Connectome imaging for mapping human brain pathways. Mol Psychiatry 2017 (04 May 2017)

8 Farquharson S, Tournier JD, Calamante F, et al. White matter fiber tractography: why we need to move beyond DTI. J Neurosurg 2013;118:1367-77.

9 Abhinav K, Yeh FC, Mansouri A, et al. High-definition fiber tractography for the evaluation of perilesional white matter tracts in high-grade glioma surgery. Neuro Oncol 2015;17:nov113-209.

10 Mandonnet E, Nouet A, Gatignol P, et al. Does the left inferior longitudinal fasciculus play a role in language? A brain stimulation study. Brain 2007;130:623-9.

11 Aaronson NK, Taphoorn MJ, Heimans JJ, et al. Compromised health-related quality of life in patients with low-grade glioma. J Clin Oncol 2011;29:4430-5.

12 Pujol S, Wells W, Pierpaoli C, et al. The DTI challenge: toward standardized evaluation of diffusion tensor imaging tractography for neurosurgery. J Neuroimaging 2015;25:875-82.
13 Bürgel U, Amunts $\mathrm{K}$, Hoemke $\mathrm{L}$, et al. White matter fiber tracts of the human brain: three-dimensional mapping at microscopic resolution, topography and intersubject variability. Neuroimage 2006;29:1092-105.

14 Bucci M, Mandelli ML, Berman Jl, et al. Quantifying diffusion MRI tractography of the corticospinal tract in brain tumors with deterministic and probabilistic methods. Neuroimage Clin 2013;3:361-8.

15 Frey D, Strack V, Wiener E, et al. A new approach for corticospinal tract reconstruction based on navigated transcranial stimulation and standardized fractional anisotropy values. Neuroimage 2012;62:1600-9.

16 Weiss Lucas C, Tursunova I, Neuschmelting V, et al. Functional MRI vs. navigated TMS to optimize M1 seed volume delineation for DTI tractography. A prospective study in patients with brain tumours adjacent to the corticospinal tract. Neuroimage Clin 2017:13:297-309.

17 Wu JS, Zhou LF, Tang WJ, et al. Clinical evaluation and follow-up outcome of diffusion tensor imaging-based functional neuronavigation: a prospective, controlled study in patients with gliomas involving pyramidal tracts. Neurosurgery 2007;61:935-48.

18 Schmahmann JD, Pandya DN. Fiber Pathways of the brain. New York: Oxford University Press, Inc, 2006.

19 Makris N, Kennedy DN, Mclnerney S, et al. Segmentation of subcomponents within the superior longitudinal fascicle in humans: a quantitative, in vivo, DT-MRI study. Cereb Cortex 2005;15:854-69.

20 Lawes IN, Barrick TR, Murugam V, et al. Atlas-based segmentation of white matter tracts of the human brain using diffusion tensor tractography and comparison with classical dissection. Neuroimage 2008;39:62-79.

21 Caverzasi E, Hervey-Jumper SL, Jordan KM, et al. Identifying preoperative language tracts and predicting postoperative functional recovery using HARDI q-ball fiber tractography in patients with gliomas. J Neurosurg 2016;125:33-45.

22 Rolheiser T, Stamatakis EA, Tyler LK. Dynamic processing in the human language system: synergy between the arcuate fascicle and extreme capsule. J Neurosci 2011;31:16949-57.

23 Marchina S, Zhu LL, Norton A, et al. Impairment of speech production predicted by lesion load of the left arcuate fasciculus. Stroke 2011:42:2251-6.

24 Poeppel D, Emmorey K, Hickok G, et al. Towards a new neurobiology of language. J Neurosci 2012:32:14125-31.

25 Vergani F, Lacerda L, Martino J, et al. White matter connections of the supplementary motor area in humans. J Neurol Neurosurg Psychiatry 2014;85:1377-85.

26 Duffau H, Gatignol P, Mandonnet E, et al. New insights into the anatomofunctional connectivity of the semantic system: a study using cortico-subcortical electrostimulations. Brain 2005;128:797-810.

27 Forkel SJ, Thiebaut de Schotten M, Kawadler JM, et al. The anatomy of fronto-occipital connections from early blunt dissections to contemporary tractography. Cortex 2014:56:73-84.

28 Bello L, Gambini A, Castellano A, et al. Motor and language DTI fiber tracking combined with intraoperative subcortical mapping for surgical removal of gliomas. Neuroimage 2008;39:369-82.

29 Harvey DY, Schnur TT. Distinct loci of lexical and semantic access deficits in aphasia: Evidence from voxel-based lesion-symptom mapping and diffusion tensor imaging. Cortex 2015;67:37-58.

30 Catani M, Jones DK, Donato R, et al. Occipito-temporal connections in the human brain. Brain 2003;126:2093-107

31 Epelbaum S, Pinel P, Gaillard R, et al. Pure alexia as a disconnection syndrome: new diffusion imaging evidence for an old concept. Cortex 2008;44:962-74.

32 Greenblatt SH. Alexia without agraphia or hemianopsia. Anatomical analysis of an autopsied case. Brain 1973:96:307-16.

33 Gil-Robles S, Carvallo A, Jimenez MM, et al. Double dissociation between visual recognition and picture naming: a study of the visual language connectivity using tractography and brain stimulation. Neurosurgery 2013;72:678-86.

34 Martino J, De Witt Hamer PC, Berger MS, et al. Analysis of the subcomponents and cortical terminations of the perisylvian superior longitudinal fasciculus: a fiber dissection and DTI tractography study. Brain Struct Funct 2013;218:105-21.

35 De Witt Hamer PC, Moritz-Gasser S, Gatignol P, et al. Is the human left middle longitudinal fascicle essential for language? A brain electrostimulation study. Hum Brain Mapp 2011;32:962-73.

36 Yourganov G, Fridriksson J, Rorden C, et al. Multivariate Connectome-based symptom mapping in post-stroke patients: networks supporting language and speech. J Neurosci 2016;36:6668-79.

37 Thiebaut de Schotten M, Urbanski M, Duffau H, et al. Direct evidence for a parietalfrontal pathway subserving spatial awareness in humans. Science 2005;309:2226-8.

38 Ebeling U, Reulen HJ. Neurosurgical topography of the optic radiation in the temporal lobe. Acta Neurochir 1988:92:29-36.

39 Sherbondy AJ, Dougherty RF, Napel S, et al. Identifying the human optic radiation using diffusion imaging and fiber tractography. J Vis 2008:8:12-11.

40 Bürgel U, Schormann T, Schleicher A, et al. Mapping of histologically identified long fiber tracts in human cerebral hemispheres to the MRI volume of a reference brain: position and spatial variability of the optic radiation. Neuroimage 1999;10:489-99. 
41 Kamada K, Todo T, Morita A, et al. Functional monitoring for visual pathway using real-time visual evoked potentials and optic-radiation tractography. Neurosurgery 2005;57:121-7. discussion 21-7.

42 Piper RJ, Yoong MM, Kandasamy J, et al. Application of diffusion tensor imaging and tractography of the optic radiation in anterior temporal lobe resection for epilepsy: a systematic review. Clin Neurol Neurosurg 2014;124:59-65.

43 Winston GP, Daga P, White MJ, et al. Preventing visual field deficits from neurosurgery. Neurology 2014;83:604-11.

$44 \mathrm{Ma} \mathrm{R}$, Livermore LJ, Plaha P. Fast track recovery program after endoscopic and awake intraparenchymal brain tumor surgery. World Neurosurg 2016;93:246-52.

45 Patel P, Patel NV, Danish SF. Intracranial MR-guided laser-induced thermal therapy: single-center experience with the Visualase thermal therapy system. J Neurosurg 2016;125:853-60.

46 Sloan AE, Ahluwalia MS, Valerio-Pascua J, et al. Results of the NeuroBlate system firstin-humans phase I clinical trial for recurrent glioblastoma: clinical article. J Neurosurg 2013; 118:1202-19.

47 Greene-Schloesser D, Robbins ME. Radiation-induced cognitive impairment--from bench to bedside. Neuro Oncol 2012;14:iv37-iv44.

48 Hope TR, Vardal J, Bjørnerud A, et al. Serial diffusion tensor imaging for early detection of radiation-induced injuries to normal-appearing white matter in highgrade glioma patients. J Magn Reson Imaging 2015;41:414-23.

49 Connor M, Karunamuni R, McDonald C, et al. Dose-dependent white matter damage after brain radiotherapy. Radiother Oncol 2016;121:209-16.

50 Chapman CH, Zhu T, Nazem-Zadeh M, et al. Diffusion tensor imaging predicts cognitive function change following partial brain radiotherapy for low-grade and benign tumors. Radiother Oncol 2016;120:234-40.

51 Maruyama K, Koga T, Kamada K, et al. Arcuate fasciculus tractography integrated into Gamma Knife surgery. J Neurosurg 2009;111:520-6.
52 Munck Af Rosenschöld P, Engelholm S, Ohlhues L, et al. Photon and proton therapy planning comparison for malignant glioma based on CT, FDG-PET, DTI-MRI and fiber tracking. Acta Oncol 2011; 50:777-83.

53 Conti A, Pontoriero A, Ricciardi GK, et al. Integration of functional neuroimaging in CyberKnife radiosurgery: feasibility and dosimetric results. Neurosurg Focus 2013;34:E5

54 Koga T, Shin M, Maruyama K, et al. Integration of corticospinal tractography reduces motor complications after radiosurgery. International journal of radiation oncology, biology. Physics 2012;83:129-33.

55 Li SP, Padhani AR. Tumor response assessments with diffusion and perfusion MRI. J Magn Reson Imaging 2012;35:745-63.

56 Ahles TA, Saykin AJ. Candidate mechanisms for chemotherapy-induced cognitive changes. Nat Rev Cancer 2007;7:192-201.

57 Deprez S, Amant F, Smeets A, et al. Longitudinal assessment of chemotherapy-induced structural changes in cerebral white matter and its correlation with impaired cognitive functioning. J Clin Oncol 2012;30:274-81.

58 Billiet T, Emsell L, Vandenbulcke M, et al. Recovery from chemotherapy-induced white matter changes in young breast cancer survivors? Brain Imaging Behav 2017 (20 Jan 2017).

59 Galbán CJ, Hoff BA, Chenevert TL, et al. Diffusion MRI in early cancer therapeutic response assessment. NMR Biomed 2017;30:e3458.

60 Duffau H, Taillandier L. New concepts in the management of diffuse low-grade glioma: proposal of a multistage and individualized therapeutic approach. Neuro Oncol 2015; 17:332-42.

61 Forkel SJ, Thiebaut de Schotten M, Dell'Acqua F, et al. Anatomical predictors of aphasia recovery: a tractography study of bilateral perisylvian language networks. Brain 2014;137:2027-39. 


\section{Brain white matter fibre tracts: a review of functional neuro-oncological relevance}

Natalie L Voets, Andreas Bartsch and Puneet Plaha

J Neurol Neurosurg Psychiatry 2017 88: 1017-1025 originally published online July 14, 2017

doi: 10.1136/jnnp-2017-316170

Updated information and services can be found at:

http://jnnp.bmj.com/content/88/12/1017

\section{These include:}

References This article cites 56 articles, 9 of which you can access for free at: http://jnnp.bmj.com/content/88/12/1017\#BIBL

Email alerting Receive free email alerts when new articles cite this article. Sign up in the service box at the top right corner of the online article.

Topic Articles on similar topics can be found in the following collections Collections

$$
\text { Neurosurgery (1) }
$$

\section{Notes}

To request permissions go to:

http://group.bmj.com/group/rights-licensing/permissions

To order reprints go to:

http://journals.bmj.com/cgi/reprintform

To subscribe to BMJ go to:

http://group.bmj.com/subscribe/ 